\title{
Optimal Placement of Distribution Generation in Radial Distribution System Using Hybrid Genetic Dragonfly Algorithm
}

\author{
G. V. Naga Lakshmi ${ }^{1}$ A. Jayalaxmi ${ }^{2}$. Venkataramana Veeramsetty ${ }^{3}$ (D)
}

Received: 4 August 2020 / Accepted: 5 May 2021 / Published online: 15 June 2021

(C) The Author(s), under exclusive licence to Springer Nature Singapore Pte Ltd. 2021

\begin{abstract}
Optimal placement of distributed generation (DG) is an essential task for distribution companies in order to operate the distribution network in good operating conditions. Optimal placement of DG units is an optimization problem where minimization of active power losses in the network is considered as an objective function. In this paper, hybrid genetic dragonfly algorithm is used as an optimization technique to find the optimal location and size of distributed generation units. The proposed algorithm is implemented on IEEE 15 and PG \& E 69 bus distribution systems in MATLAB environment. Based on the simulation results it has been observed that with proper placement and size of DG units, distribution network can be operated with less active power losses.
\end{abstract}

Keywords Distributed generation · Optimal placement $\cdot$ Hybrid genetic dragonfly algorithm $\cdot$ Active power loss

\section{Nomenclature}

$\Delta Y_{t+1} \quad$ Step vector for dragonflies

$A^{i} \quad$ Alignment value of dragonfly $i$

$C^{i} \quad$ Cohesion value of dragonfly $i$

$E^{i} \quad$ Enemy source of dragonfly $i$

$F^{i} \quad$ Food source of dragonfly $i$

$I_{i}^{k} \quad$ Current at bus ' $\mathrm{i}$ ' and iteration ' $\mathrm{k}$ '

$I_{L R}^{k} \quad$ Current injecting at receiving node of line ' $\mathrm{L}$ ' at iteration ' $\mathrm{k}$ '

$I_{l} \quad$ Current flowing through line $l$

$I_{L}^{k} \quad$ Current through line ' $\mathrm{L}$ ' at iteration ' $\mathrm{k}$ '

$I_{l}^{\max } \quad$ Maximum allowable current flowing through line $l$

loc Location for DG units

Venkataramana Veeramsetty

dr.vvr.research@gmail.com

G. V. Naga Lakshmi

nagalakshmi.eedou@gmail.com

A. Jayalaxmi

aj11994@yahoo.co.in

1 Department of Electrical Engineering, Osmania University, Hyderabad, Telangana, India

2 Department of Electrical and Electronics Engineering, JNTU Hyderabad, Hyderabad, Telangana, India

3 Department of Electrical and Electronics Engineering, Center for Artificial Intelligence and Deep Learning,

S R Engineering College, warangal, Telangana, India
$L R \quad$ Receiving node of line ' $\mathrm{L}$ '

$n \quad$ Number of buses

$n_{\text {line }} \quad$ Number of lines in distribution network

$P_{g} \quad$ Active power generation of DG $g$

$P_{g}^{\max } \quad$ Maximum active power generation of DG $g$

$P_{g}^{\min } \quad$ Minimum active power generation of DG $g$

$P_{\text {loss }} \quad$ Active power losses

$R_{l} \quad$ Resistance of line 1

$S^{i} \quad$ Separation value of dragonfly $i$

$S_{i} \quad$ Complex power injection at bus ' $\mathrm{i}$ '

$V_{i} \quad$ Magnitude of voltage at bus $i$

$V_{i}^{k-1} \quad$ Complex voltage at bus ' $\mathrm{i}$ ' and iteration ' $\mathrm{k}-1$ '

$V_{i}^{\max } \quad$ Maximum bus voltage at bus $i$

$V_{i}^{\text {min }} \quad$ Minimum bus voltage at bus $i$

$V_{L R}^{k}$

$V_{L R}^{k}$ Voltage at receiving end node of line ' $L$ ' at iteration ' $\mathrm{k}$ '

$V_{L S}^{k} \quad$ Voltage at sending end node of line ' $\mathrm{L}$ ' at iteration ' $\mathrm{k}$ '

$V_{l}^{R} \quad$ Receiving end node for line 1

$V_{l}^{S} \quad$ Sending end node for line 1

$Y^{+} \quad$ Position of food

$Y^{-} \quad$ Position of enemy

$Y_{i} \quad$ Sum of all shunt admittences at bus 'i'

$Y_{j} \quad$ Position of neighborhood dragonfly

$Z_{L} \quad$ Impedance of line ' $L$ '

$Z_{l} \quad$ Impedance of line 1

a Weight corresponding to Alignment

c Weight corresponding to Cohesion 
e Weight corresponding to Enemy

f Weight corresponding to Food

M Number of dragonflies in neighborhood

s Weight corresponding to Separation

Y Position of current dragonfly

\section{Introduction}

Distributed Generation (DG) integration into the distribution system has been growing very fast due to technical, economical and environmental benefits $[1,2]$ as presented in Fig. 1. The DG integration may have its positive or negative impact on the distribution system, depending upon the location and size of DG. The positive benefits built into the distribution network are accomplished by putting the DG in an optimal position and size. The incorrect position of DG has a detrimental impact on the distribution network.

Generally, distribution networks are in radial stricture with high $\frac{R}{X}$ value that leads more power losses than in transmission system which have low $\frac{R}{X}$ value. The superior problem in electric power distribution systems is high power losses due to which $13 \%$ of generated power is wasted in distribution networks [3]. The need for solving this problem is an open research area with the aim of finding new methodologies. In this scenario, this paper proposes a new hybrid genetic dragonfly algorithm methodology that finds the optimal location and size of the DG units to enhance the active power losses of distribution systems. Optimal capacitor placement can also be used to minize the active power losses in the system [4].

Many studies that are available in the literature have proposed various optimization algorithms and objective functions to find the optimal placement and size of DG units so that distribution companies will get positive benefits with integration of DG units into the distribution system. This research problem was attempted in [5] as weighted multiobjective optimization problem by considering the active and reactive power losses of the system, voltage profile,line loading, and the voltage stability as objectives. Authors have used improved raven roosting optimization (IRRO) algorithm has been implemented for optimal deployment of DG in RDS. The proposed methodology was implemented on IEEE-33 and PG \& E 69 bus systems. Enhanced Genetic Algorithm was developed in [6] for optimal placement of DG units and capacitors in RDS. Minimization of total active power losses of the system and voltage deviation were are considered as objectives for this optimization problem. The proposed methodology is implemented on IEEE 33, PG \& E 69 and 119 bus systems.

A new methodology is developed in [7] for optimal placement of DG units using fuzzy logic controller, Ant-lion optimization algorithm and particle swarm optimization. Minimization of power losses and voltage deviation have been considered as objectives. The proposed technique was simulated on IEEE 33 bus test system. A novel scheme based on quasi-oppositional chaotic symbiotic organisms search (QOCSOS) algorithm is developed in [8] to find the optimal number of DG penetrations. The proposed methodology is simulated on IEEE 33, 69, and 118-bus test networks. Voltage stability index and whale optimization algorithm based methodology is developed in [9] for optimal placement of DG units. VSI is used to identify the most sensitive bused to connect DG units. The proposed methodology is tested on 12, 33, 69 and 85 bus distribution networks.

Optimal placement of DG units by considering the minimization of losses and maximization loading capability is addressed in [10]. Authors have used Flower Pollination Algorithm to solve this optimization problem. The proposed method is implemented on IEEE 33 bus and PG \& E 69 bus test systems. Solution for optimal placement of DG based

Fig. 1 Benefits with DG integration

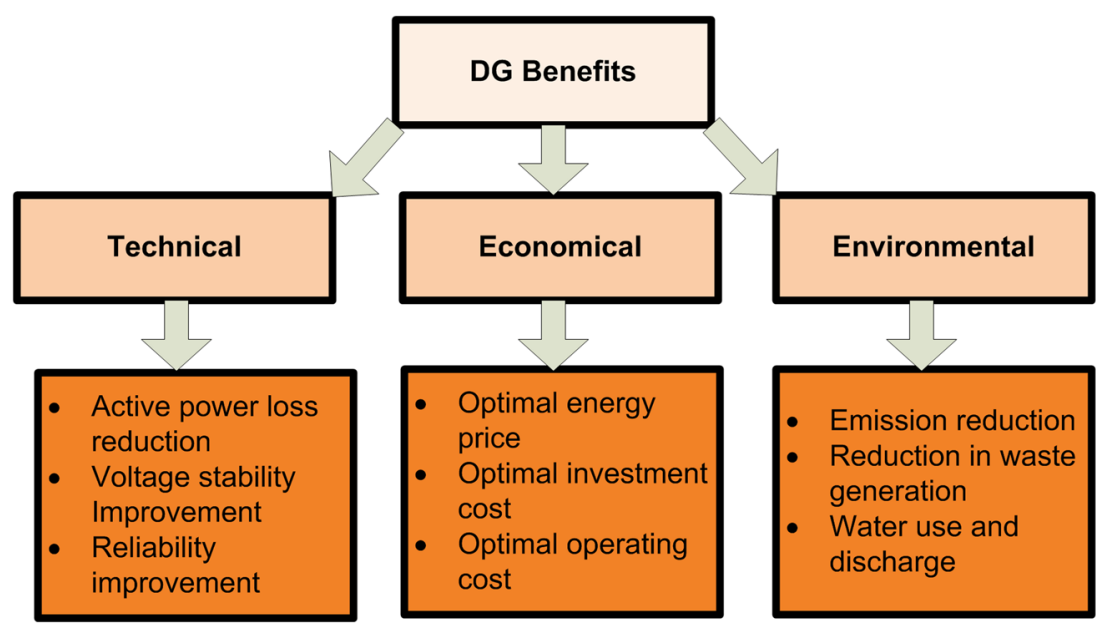


on firefly algorithm is discussed in [11]. Authors have considered minimization of active power losses as an objective and tested on IEEE 33 and PG\& E 69 bus distribution systems. Fuzzy expert system based approach was developed in [12] for optimal placement of DG units. Optimal placement was identified based on Distribution Loss Reduction Index (DLRI) and Voltage Deviation Reduction Index (VDRI). In addition to these some more literature is available for optimal placement of DG units in radial distribution system (RDS)as shown in Table 1. All the methodologies provide valuable contribution for this research problem.

In this paper hybrid genetic dragonfly based metaheuristic algorithm is developed and used for the first time to solve optimal DG placement problem. Minimization of active power losses is considered as an objective function. The proposed optimization problem will help the distribution network operator to operate the network with good voltage stability as voltage limits included in the optimization problem as inequality constraints. This methodology also helps the distribution network operator to operate the network without violating the thermal limits of the feeders as thermal line limits have included in the optimization problem as another inequality constraints. Location interms of bus number in distribution system and size of DG in terms of generating capacity are considered as decision variables.

The remaining part of the paper has been organized as follows: "Methodology" describes about problem formulation, "Analytical Study" reports simulation results of the proposed method and "Conclusions" summarizes the conclusions of this paper.

\section{Methodology}

This section presents mathematical modeling of optimization problem related to optimal placement of DG units, describes the hybrid meta-heuristic algorithm and distribution load flow algorithm. Inequality constraints of this optimization problem are presented in Eq. 2.

\section{Mathematical Modeling of DG Placement Optimization Problem}

Mathematical modeling of DG placement optimization problem consists minimization of active power losses in distribution network as objective function as shown in Eq. 1.

$P_{\text {loss }}=\operatorname{Real}\left[\sum_{l=1}^{n_{\text {line }}}\left(\frac{\left|V_{l}^{S}-V_{l}^{R}\right|}{Z_{l}}\right)^{2} R_{l}\right]$

The inequality constraints of the problem under study are voltage constraints, line thermal limits constraints. Voltage constraints defines the voltage magnitude of a node should lie within voltage and lie between the range from the minimum voltage $\left(V_{\min }\right)$ to maximum voltage $\left(V_{m} a x\right)$ as shown in Eq. 2.

$V_{i}^{\min } \leq V_{i} \leq V_{i}^{\max }$

The current $I_{l}$ through any line ' $l$ ' is must be less than the maximum current capacity of this branch $I_{l}^{\max }$ as shown in Eq. 3.

$I_{l} \leq I_{l}^{\max }$
Table 1 Literature report on optimal placement of EG units in distribution system

\begin{tabular}{llll}
\hline Reference & Bus information & Objectives & Algorithm \\
\hline Kim et al [13] & $6,14,30$ & Minimization of losses & GA \\
Gandomkar et al [14] & 33,69 & Minimization of losses & GA \\
Haesen et al [15] & 69 & Minimization of losses & GA \\
Singh and Goswami [16] & 33 & Minimization of losses & GA \\
Singh et al [17] & 16,37 & Minimization of losses & GA \\
Veeramsetty et al [18] & 69 & Minimization of losses & GA \\
Prommee and Ongsakul [19] & 69 & Minimization of energy losses & PSO \\
El-Zonkoly [20] & 30 & Minimization of power losses & PSO \\
Wang and Singh [21] & 13,33 & Multiple objectives & ACO \\
Abu-Mouti and El-Hawary [22] & 69 & Maximize voltage limit & ABC \\
Rao et al [23] & 33,69 & Maximize EG Capasity & HS \\
Ghosh et al [24] & $6,14,30$ & Maximize EG Capasityt & PH \\
Koutroumpezis and Safigianni [25] & 31 & Minimization of energy losses & PH \\
Mohd Ikhwan et al [26] & $11 / 0.4 \mathrm{kV}$ & Reliability improvement & MCS \\
Lakshmi et al [27] & 15,69 & Minimization of power losses & FA \\
Fathi and Ghiasi [28] & 33 & Minimum DG investment cost & GA, PSO \\
\hline
\end{tabular}


Generation capacity of DG unit i.e. $P_{g}$ and location are the decision variables. The boundary values for these two decision variables are presented in Eq. 4

$$
\begin{aligned}
P_{g}^{\min } \leq & P_{g} \leq P_{g}^{\max } \\
& 2<l o c<n
\end{aligned}
$$

\section{Genetic Algorithm}

Genetic Algorithm (GA) was first introduced by John Holland and further developed by Goldberg [29]. In GA an array of all control variables is represented by chromosomes and the number of chromosomes generated depends on population size. New generations have been evaluated from old generations using genetic operators like reproduction, cross over and mutation. Elitism operator has been used to keep better individuals of previous generation. Real coded genetic algorithm has been used in this paper. The values of GA parameters considered in this paper are as represented in Table 2.

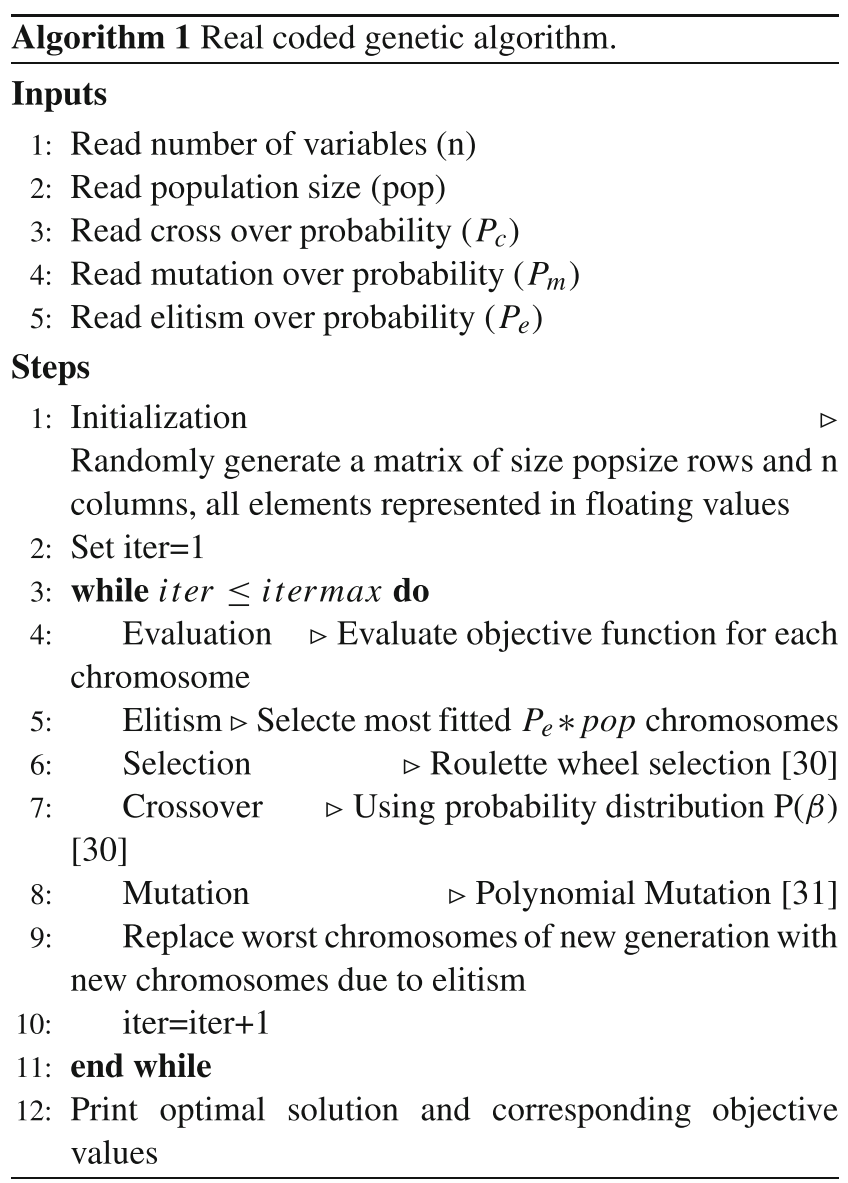

\section{Dragonfly Algorithm (DA)}

The existing conventional algorithms have drawbacks such as a highly responsive starting point and sometimes converge
Table 2 The values of GA parameters

\begin{tabular}{ll}
\hline Parameter & Value \\
\hline Control Varible & $P_{g}$, loc \\
Population & 80 \\
Cross over probability $\left(P_{c}\right)$ & 0.9 \\
Mutation probability $\left(P_{m}\right)$ & 0.01 \\
Elitism probability $\left(P_{m}\right)$ & 0.05 \\
Maximum iterations & 100 \\
\hline
\end{tabular}

to a local optimum solution. Bio-inspired algorithms have been developed to solve the problem specific issues [3234]. Dragonfly Algorithm (DA) was one of the bio-inspired algorithms that evolved based on dragonflies swarming behavior.

Two types of swarming that exists are static and dynamic. Dragonflies form small groups and move forward and backward over a small region to hunt other flying preys during static swarm. Whereas, during dynamic swarm, large number of dragonflies moves in one direction over a long distance for migration. Dynamic swarming with high alignment and low cohesion is related to exploration phase and static swarming with low alignment and high cohesion can be related to exploitation in optimization problem. The five main factors considered in swarming of dragonflies are separation, alignment, cohesion, attracting towards food and detracting away from enemy. The above factors have been mathematically modeled in Eqs. 5, 6, 7, 8 and 9 respectively [35]. The values of DA parameters considered in this paper are as represented in Table 3.

$S^{i}=-\sum_{i=1}^{M} Y-Y_{i}$

$A^{i}=\frac{\sum_{j=1}^{N} Y_{j}}{N}$

$C^{i}=\frac{\sum_{j=1}^{N} Y_{j}}{N}-Y$

Table 3 The values of DA parameters

\begin{tabular}{ll}
\hline Parameter & Value \\
\hline Control Varible & $P_{g}$, loc \\
Population & 80 \\
Weight (w) & $0.9-0.4$ \\
Separation factor (s) & $0.2-0$ \\
Alignment factor (a) & $0.2-0$ \\
Cohesion factor (c) & $0.2-0$ \\
Food factor (f) & $0-2$ \\
Enemy factor (e) & $0.1-0$ \\
Maximum iterations & 100 \\
\hline
\end{tabular}


$F^{i}=Y^{+}-Y$

$E^{i}=Y^{-}+Y$

As per dragonfly algorithm step of dragonflies is updated using Eq. 10. If neighborhood exist then position of dragonflies is updated using Eq. 11 otherwise using Eq. 12. Complete flowchart for solving any optimization problem using dragonfly algorithm is shown in Fig. 2.

$$
\begin{aligned}
& \Delta Y_{t+1}=\left(s S^{i}+a A^{i}+c C^{i}+f F^{i}+e E^{i}\right)+w \Delta Y_{t} \\
& Y_{t+1}=Y_{t}+\Delta Y_{t+1} \\
& Y_{t+1}=Y_{t}+\text { Levy } * Y_{t}
\end{aligned}
$$

\section{Hybrid Genetic Dragonfly Algorithm (HGDA)}

In order to accomplish a global optimum with any populationbased algorithm, a proper balance is needed among exploration and exploitation of the search area. Exploration associated with global solution space and exploitation related to local solution space are based on the current best solution. Very much diversification and intensification would result in an increase in the period of convergence and an increase in the probability of a solution getting stuck at the local equilibrium point [36].

Genetic Algorithm has a difficulty identifying an accurate solution, but it is useful to enter a global area and gradual convergence. GA operates on the principle of adaptation

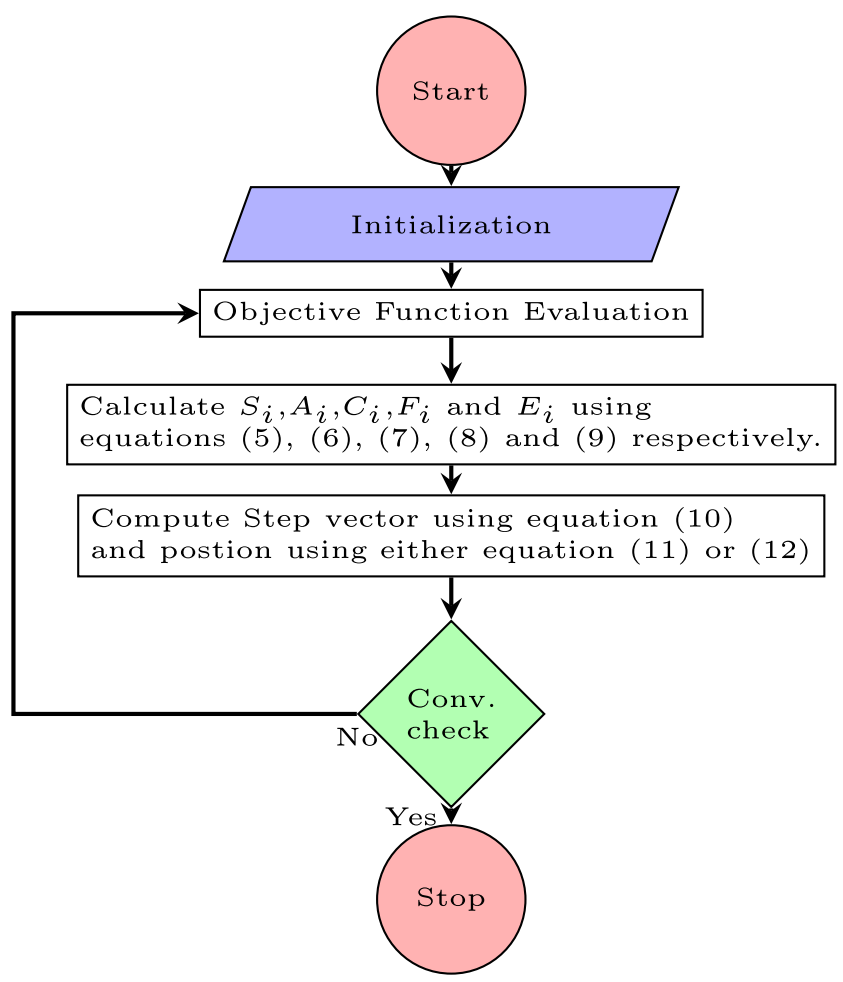

Fig. 2 Dragonfly Algorithm [35] from generation to generation by not considering individuals in the same group. Simple GA has no memory, which ensures that prior experience of the problem is lost if the population shifts [37]. But this question can be solved by introducing the idea of elitism. This means that the GA idea of elitism has a history that better preserves individuals from the previous community.

However, in comparison to GA, DA has quick convergence and quality of algorithm is improved when best individuals are available, but it does not have internal memory. Owing to lack of internal memory, DA rarely keeps track of a conceivable collection of solutions that have the ability to converge to the global optimum, which may contribute to the solution being stuck towards the local optimum point [38].

To address the disadvantages described above, a novel hybrid algorithm based on GA and DA was proposed in this paper to maximize the strengths of both GA and DA algorithms. The hybridization requires two new aspects of DA, such as intrinsic memory and spontaneous search capacity. The suggested hybrid genetic dragonfly algorithm acquires sufficient local and global search capabilities to prevent the issue of limiting the solution to a particular optimum level. The step-by - step process for HGDA is shown in Fig. 3.

\section{Backward Forward Sweep Load-Flow Analysis of Radial Distribution Systems}

Conventional load flow techniques those are used in transmission system may not work well in distribution system due to high $\mathrm{R} / \mathrm{X}$ values. In this paper backward forward

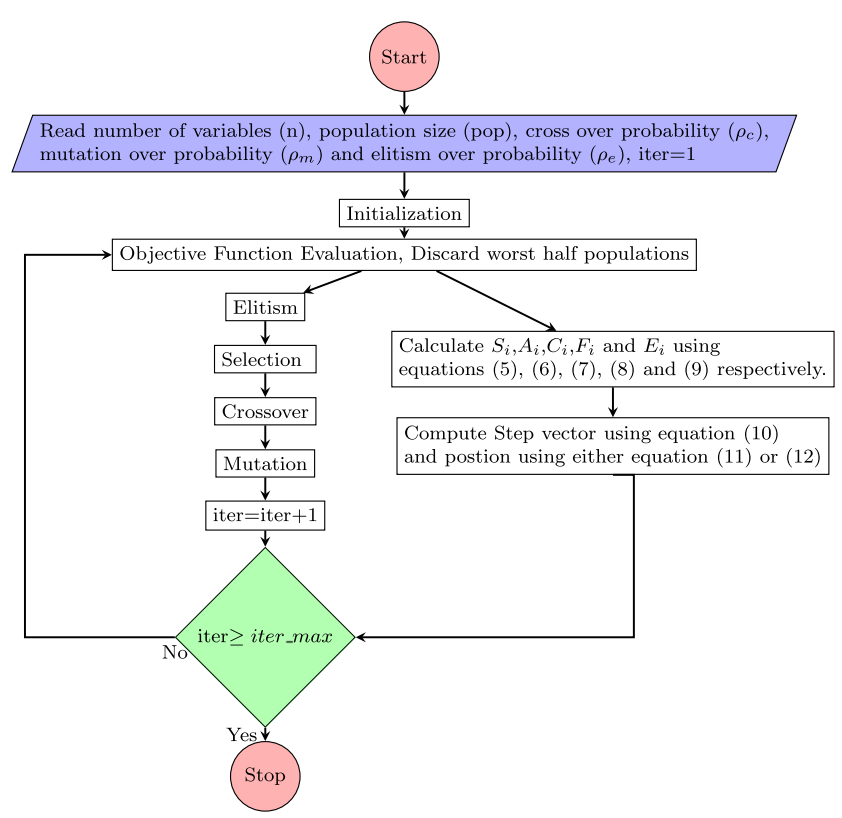

Fig. 3 Hybrid genetic dragonfly algorithm 
sweep load-flow method [39] has been used to compute voltages at each bus, active and reactive power losses of radial distribution system. This load-flow method utilizes complete advantage of ladder structure of distribution network, to achieve high speed, robust convergence and low memory requirements $[40,41]$. Step by step procedure for backward and forward load flow method is presented in flowchart in Fig. 4.

\section{Analytical Study}

The proposed method was implemented on IEEE 15 bus and PG \& E 69 bus distribution systems as shown in Figs. 5 and 6 respectively for optimally placing two DG units with 0.9 lagging power factor under MATLAB environment [42]. IEEE 15 bus and PG\& E 69 bus distribution systems data are drawn from [43].

\section{Case Study: IEEE 15 Bus Distribution System}

\section{Performance of HGDA in Stochastic Environment}

The proposed hybrid genetic dragonfly algorithm is simulated 10 times for identifying the location and generation capacity of DG units. Out of 10 simulations best one in terms of minimum active power losses is considered for identifying the location and generation capacity of DG units. Performance of the proposed algorithm is observed in stochastic environment as presented in Table 4. As the proposed algorithm provides same amount of losses over all simulations, the standard deviation is zero. Zero standard deviation represents that the proposed HGDA provides a solution without uncertainty.

Performance curves of the proposed HGDA for optimal placement of DG units over 10 simulations is presented in Fig. 7. From the Fig. 7, it has been observed that for all 10 simulations the proposed algorithm is converging at active power losses of $2600 \mathrm{~W}$.

\section{Optimal Location and Capacity for DG Units}

The optimal location and size for the two DG units in IEEE 15 bus distribution system is presented in Table 5 . From the Table 5, it has been observed that distribution network will operate with minimum losses i.e. 2600Watts if two DG units with generation capacity 476Watts and 843Watts connected at bus 9 and 4 respectively.

\section{Comparative Analysis}

The proposed HGDA for optimal placement of DG units in IEEE 15 bus distribution system is validated by comparing with Dragonfly Algorithm [35], Genetic Algorithm [29] and Particle Swarm Optimization [44]. As all these metaheuristic algorithms are stochastic in nature, comparison among all these algorithms for DG optimal placement problem is observed in stochastic environment. Each algorithm was simulated on IEEE 15 bus test system for 10 times and performance is evaluated in terms of statistical parameters like mean and standard deviation as presented in Table 6. From the Table 6, It has been observed that proposed HGDA has zero standard deviation. However remaining meta-heuristic algorithms have more standard deviation value comparing to HGDA, that shows more robust behavior of HGDA over other meta-heuristic algorithms for finding the optimal placement of DG units. The performance of proposed HGDA for optimal placement of DG has been verified statistically using wilcoxon rank sum test [45] and the obtained p-values are presented in Table 6. The proposed HGDA is more significant comparing to DA [35], GA [29] and PSO [44] as p-value is less than 0.05. Dragonfly Algorithm [35] and PSO [44] provides same minimum losses $2600 \mathrm{~kW}$ as proposed HGDA but later one statistically more significant due zero standard deviation.

Comparison of proposed HGDA with other metaheuristic algorithms in terms of convergence characteristics has been presented in Fig. 8. The proposed HGDA has smooth convergence characteristics in comparison with DA, PSO and GA. HGDA reaches optimal point i.e. minimum losses quickly than DA and PSO. However GA not able to reach the optimal point within 100 iterations.

Voltage at each bus in IEEE 15 bus distribution system is presented in Fig. 9. Voltage at each bus without any DG units in the system is low comparing to system with DG units due to reverse flow of power that leads reduction in losses and improves voltage profile. Voltage profile with HGDA, DA, PSO is better than GA and almost near to unity.

The performance of HGDA, DA and PSO is almost similar for optimal allocation of DG on IEEE 15 bus distribution system if best simulation chosen. However, HGDA outperforms remaining all algorithms when tested in stochastic environment and it is necessary as all algorithms are stochastic in nature.

\section{Case Study: PG\&E 69 Bus Distribution System}

\section{Performance of HGDA in Stochastic Environment}

The proposed hybrid genetic dragonfly algorithm is simulated 10 times for identifying the location and generation capacity of DG units. Out of 10 simulations best one in terms of minimum active power losses is considered for identifying the location and generation capacity of DG units. Performance of the proposed algorithm is observed in stochastic environment as presented in Table 7. As the 
Fig. 4 Flowchart for backward forward sweep load-flow method

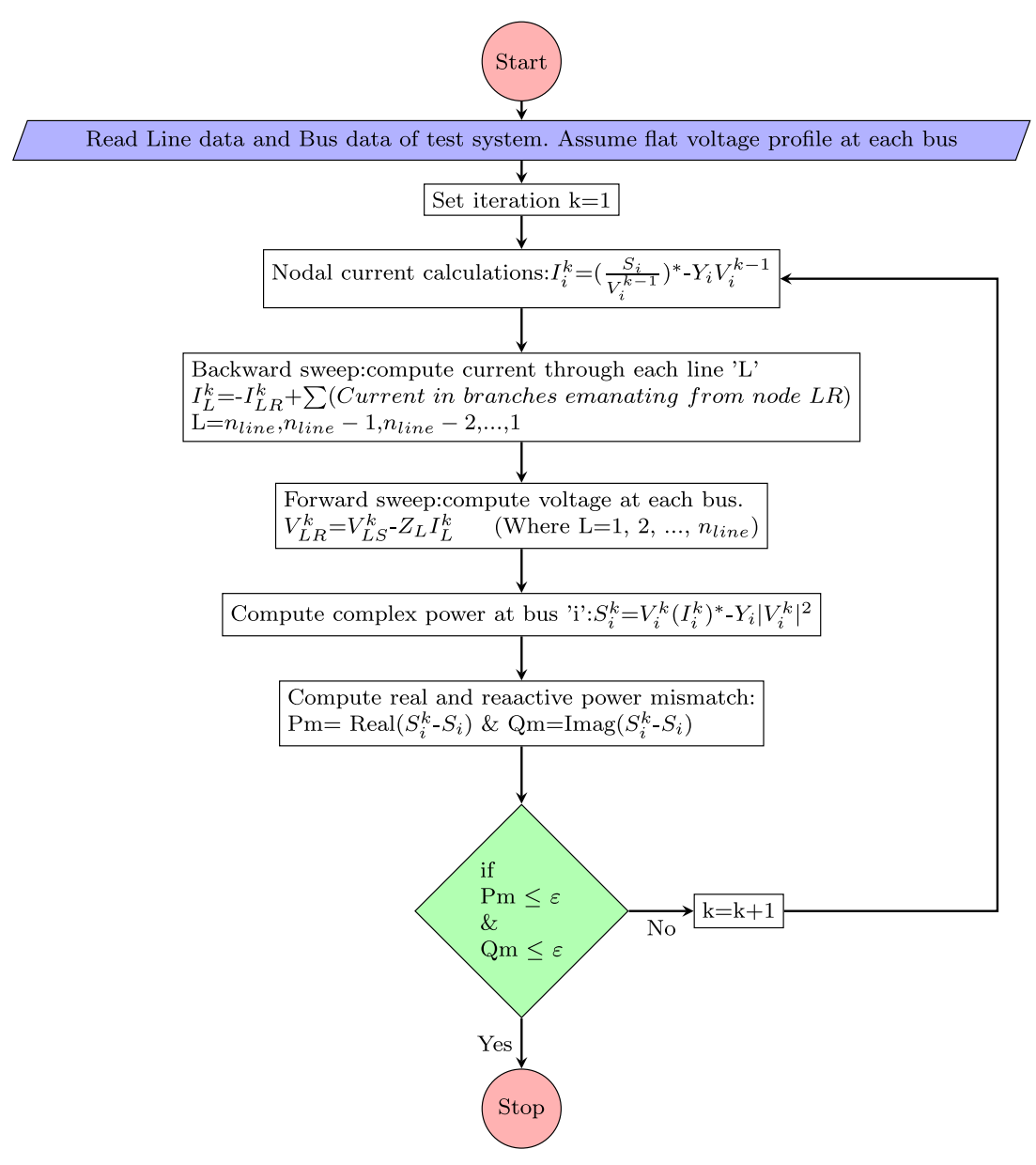

Particle Swarm Optimization [44]. As all these metaheuristic algorithms are stochastic in nature, comparison among all these algorithms for DG optimal placement

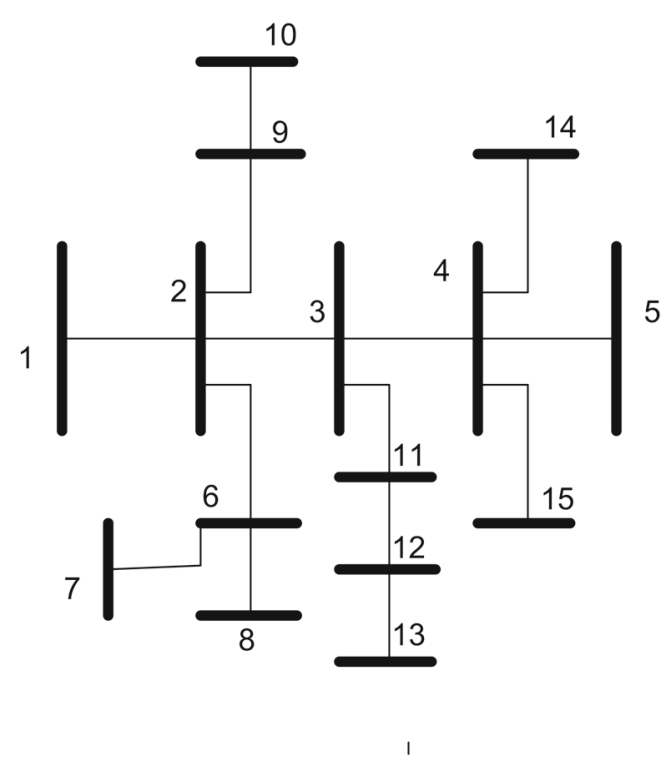

Fig. 5 Single line diagram for IEEE 15 bus distribution system [43] 
Fig. 6 Single line diagram for PG \& E 69 bus distribution system [43]

Table 4 Stochastic behavior of HGDA - IEEE 15 Bus test system

Fig. 7 Performance curves over different simulations - IEEE 15 Bus Test System

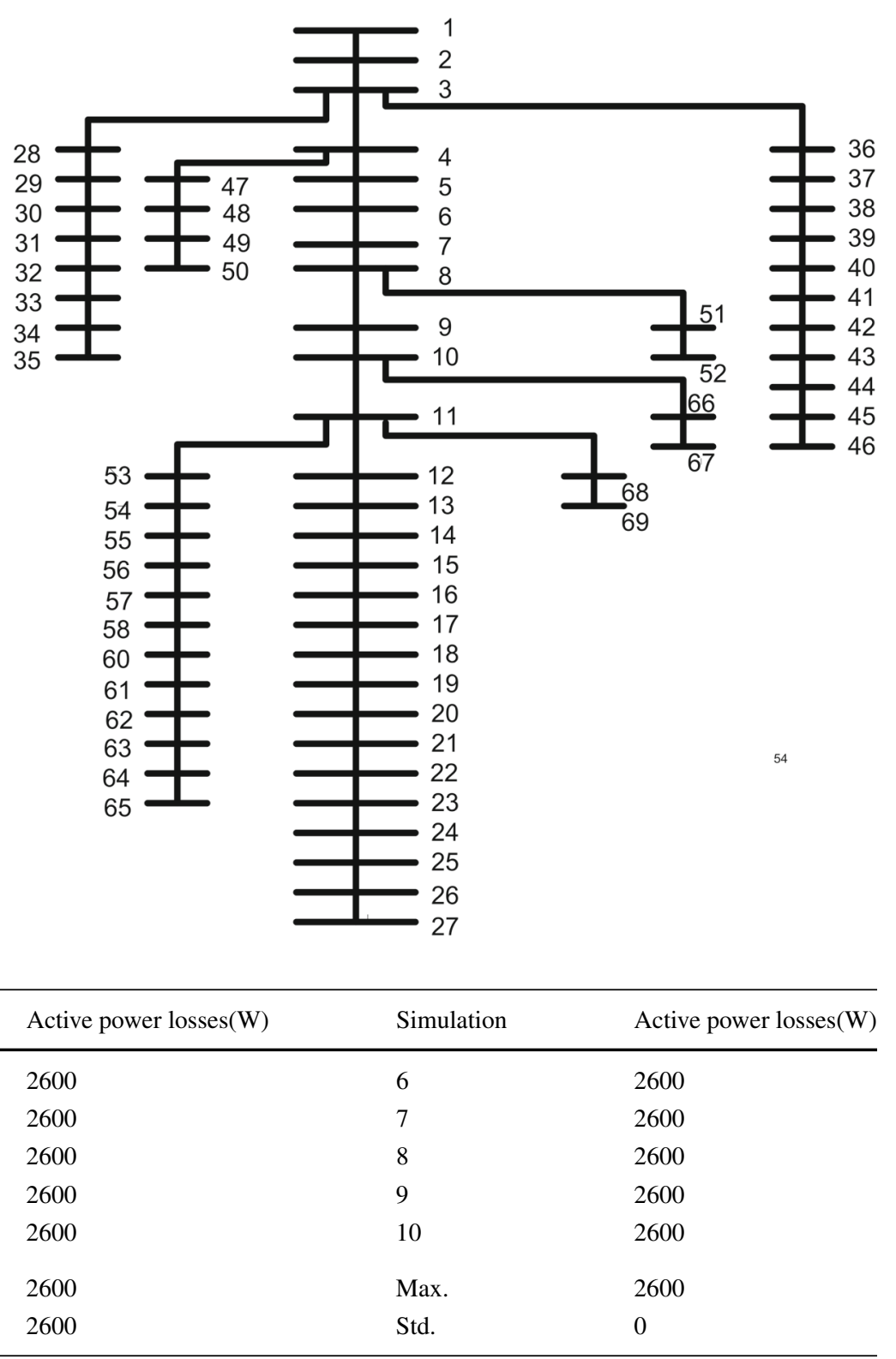

\begin{tabular}{llll}
\hline Simulation & Active power losses(W) & Simulation & Active power losses(W) \\
\hline 1 & 2600 & 6 & 2600 \\
2 & 2600 & 7 & 2600 \\
3 & 2600 & 8 & 2600 \\
4 & 2600 & 9 & 2600 \\
5 & 2600 & 10 & 2600 \\
Min. & 2600 & Max. & 2600 \\
Mean & 2600 & Std. & 0 \\
\hline
\end{tabular}


Table 5 Optimal location and size for DG units for IEEE 15

\begin{tabular}{|c|c|c|c|c|c|}
\hline \multicolumn{2}{|l|}{ DG1 } & \multicolumn{2}{|l|}{ DG2 } & \multirow[t]{2}{*}{ Loss (kW) } & \multirow{2}{*}{$\begin{array}{l}\text { Base case } \\
\text { loss }(\mathrm{kW})\end{array}$} \\
\hline Location & $\operatorname{size}(k W)$ & Location & $\operatorname{size}(k W)$ & & \\
\hline 9 & 476 & 4 & 1000 & 2.6 & 12 \\
\hline
\end{tabular}

Table 6 Comparison in terms of active power losses (Watts) in stochastic environment:IEEE 15 bus test system

\begin{tabular}{|c|c|c|c|c|c|}
\hline Simulation & Proposed Method & DA [35] & GA [29] & PSO [44] & FA [27] \\
\hline 1 & 2600 & 2880 & 3980 & 3680 & 14000 \\
\hline 2 & 2600 & 2600 & 3900 & 2600 & 16000 \\
\hline 3 & 2600 & 2600 & 5120 & 3360 & 16000 \\
\hline 4 & 2600 & 2720 & 3900 & 3000 & 15000 \\
\hline 5 & 2600 & 2720 & 3980 & 3120 & 14000 \\
\hline 6 & 2600 & 2600 & 3980 & 2740 & 15000 \\
\hline 7 & 2600 & 2880 & 3980 & 2800 & 16000 \\
\hline 8 & 2600 & 2600 & 3900 & 3140 & 18000 \\
\hline 9 & 2600 & 2720 & 3900 & 3180 & 14000 \\
\hline 10 & 2600 & 2600 & 3900 & 2720 & 14000 \\
\hline Min & 2600 & 2600 & 3900 & 2600 & 14000 \\
\hline $\operatorname{Max}$ & 2600 & 2880 & 5120 & 3680 & 18000 \\
\hline Mean & 2600 & 2692 & 4054 & 3034 & 15000 \\
\hline Std & 0 & 113.2156 & 376.657817 & 332.1379 & 1316.6 \\
\hline P-Value & NA & 0.015101 & $3.32701 \mathrm{E}-07$ & 0.001276 & $2.29 \mathrm{E}-10$ \\
\hline
\end{tabular}

Fig. 8 Convergence characteristics comparison: IEEE 15 bus test system

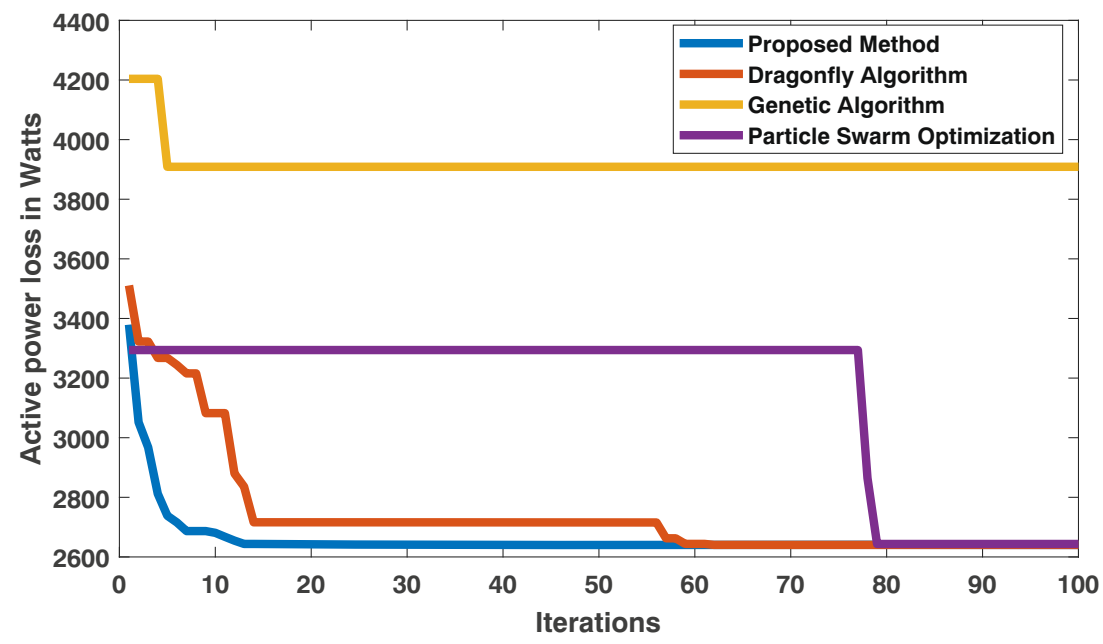


Fig. 9 Convergence

characteristics comparison:IEEE

15 bus test system

Table 7 Stochastic behavior of HGDA

Fig. 10 Performance curves over different simulations: PG\& E 69 bus test system

Table 8 Optimal location and size for DG units

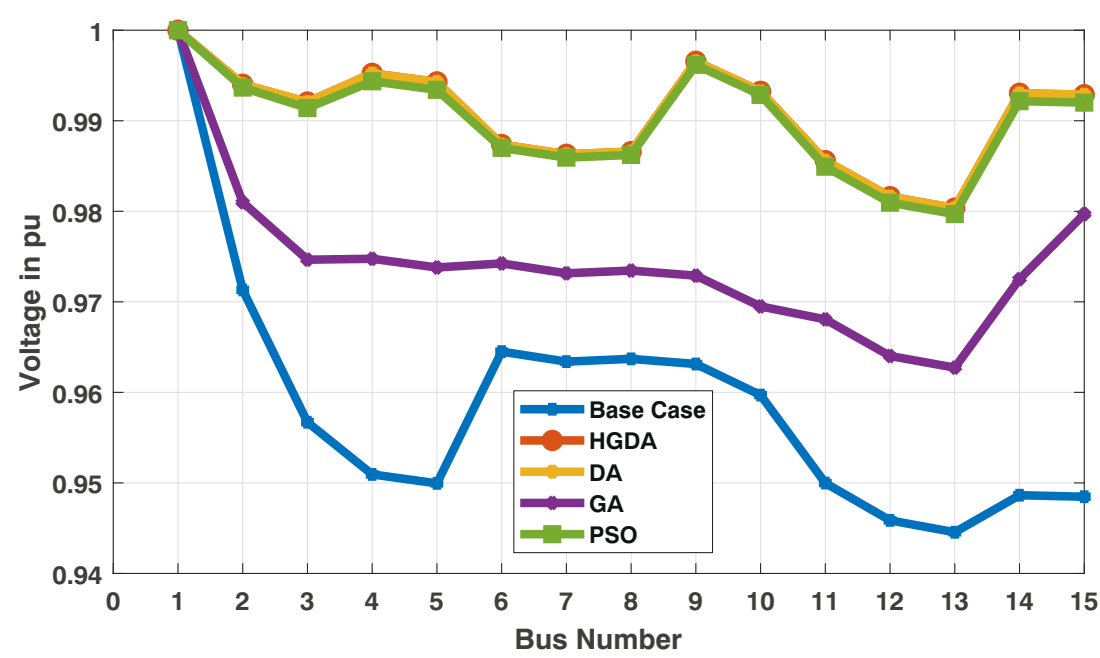

\begin{tabular}{llll}
\hline Simulation & Active power losses $(\mathrm{kW})$ & Simulation & Active power losses(kW) \\
\hline 1 & 27.9 & 6 & 27.9 \\
2 & 27.9 & 7 & 27.9 \\
3 & 27.9 & 8 & 27.9 \\
4 & 27.9 & 9 & 27.9 \\
5 & 27.9 & 10 & 27.9 \\
Min. & 27.9 & Max. & 27.9 \\
Mean & 27.9 & Std. & 0 \\
\hline
\end{tabular}

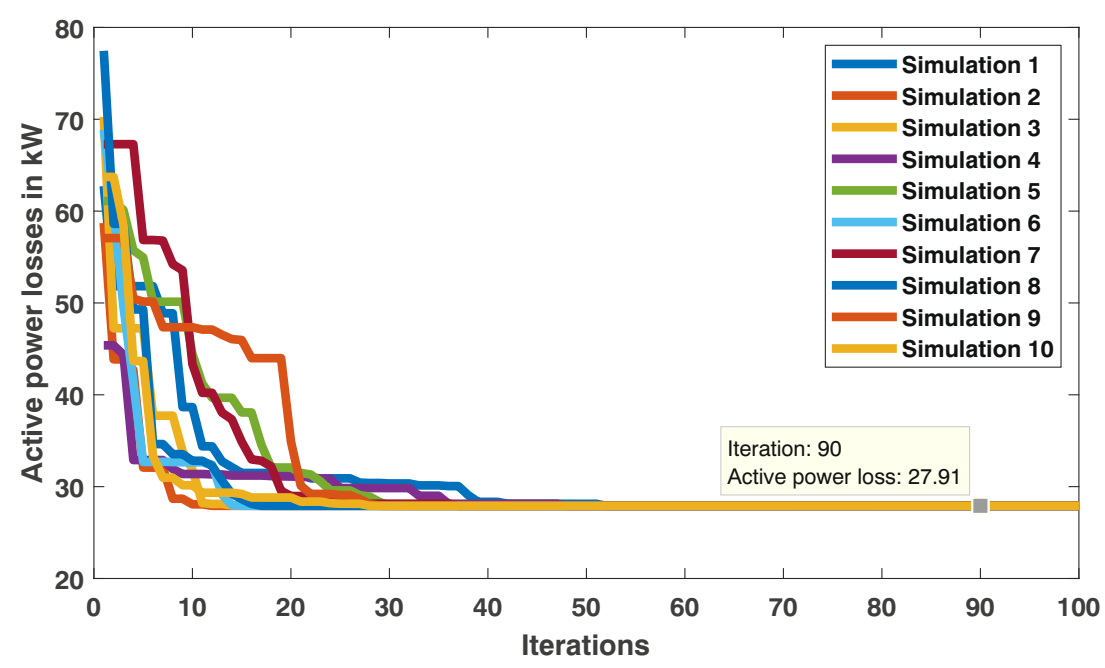

\begin{tabular}{|c|c|c|c|c|c|}
\hline \multicolumn{2}{|l|}{ DG1 } & \multicolumn{2}{|l|}{ DG2 } & \multirow[t]{2}{*}{ Loss (kW) } & \multirow{2}{*}{$\begin{array}{l}\text { Base case } \\
\text { loss }(\mathrm{kW})\end{array}$} \\
\hline Location & $\operatorname{size}(k W)$ & Location & $\operatorname{size}(\mathrm{kW})$ & & \\
\hline 61 & 1000 & 61 & 1000 & 27.9 & 221.8 \\
\hline
\end{tabular}


Table 9 Comparison of proposed method in stochastic environment

\begin{tabular}{llllll}
\hline Simulation & Proposed Method & DA [35] & GA [29] & PSO [44] & FA [27] \\
\hline 1 & 27.9 & 27.9 & 78.4 & 42.1 & 81 \\
2 & 27.9 & 30.8 & 46.1 & 33.5 & 59 \\
3 & 27.9 & 27.9 & 68.9 & 30.2 & 68 \\
4 & 27.9 & 41.0 & 95.1 & 28.6 & 81 \\
5 & 27.9 & 30.8 & 46.9 & 28.6 & 70 \\
6 & 27.9 & 27.9 & 74.3 & 49.1 & 44 \\
7 & 27.9 & 27.9 & 72.2 & 49.1 & 48 \\
8 & 27.9 & 27.9 & 72.3 & 49.1 & 57 \\
9 & 27.9 & 44.0 & 73.2 & 47.7 & 84 \\
10 & 27.9 & 31.2 & 58.8 & 37.0 & 63 \\
Min & 27.9 & 27.9 & 46.1 & 28.6 & 44 \\
Max & 27.9 & 44.0 & 95.1 & 49.1 & 84 \\
Mean & 27.9 & 31.7 & 68.6 & 39.5 & 65 \\
Std. & 0 & 5.9 & 14.7 & 8.9 & 14 \\
Time (Sec) & 185 & 102 & 170 & 78 & 555 \\
P-Value & NA & 0.034877 & $5.40282 \mathrm{E}-06$ & 0.001318 & $1.29 \mathrm{E}-05$ \\
\hline
\end{tabular}

problem is observed in stochastic environment. Each algorithm was simulated on PG\& E 69 bus test system for 10 times and performance is evaluated in terms of statistical parameters like mean and standard deviation as presented in Table 9. From the Table 9, It has been observed that proposed HGDA has zero standard deviation. However remaining meta-heuristic algorithms have more standard deviation value comparing to HGDA, that shows more robust behavior of HGDA over other meta-heuristic algorithms for finding the optimal placement of DG units. The performance of proposed HGDA for optimal placement of DG has been verified statistically using wilcoxon rank sum test [45] and the obtained p-values are presented in Table 9. The proposed HGDA is more significant comparing to DA [35], GA [29] and PSO [44] as p-value is less than 0.05. Dragonfly
Algorithm [35] provides same minimum losses $27.9 \mathrm{~kW}$ as proposed HGDA but later one statistically more significant due zero standard deviation.

Comparison of proposed HGDA with other meta-heuristic algorithms in terms of convergence characteristics has been presented in Fig. 11. Proposed HGDA and DA performing almost similar after few iterations but later one not good statistically. Proposed HGDA converging fast and provides minimum losses in comparison with GA and PSO.

Voltage at each bus in PG\& E 69 bus distribution system is presented in Fig. 12. Voltage at each bus without any DG units in the system is low comparing to system with DG units due to reverse flow of power that leads reduction in losses and improves voltage profile. Voltage profile with HGDA and DA is better than GA and PSO and almost near to unity.
Fig. 11 Convergence characteristics comparison

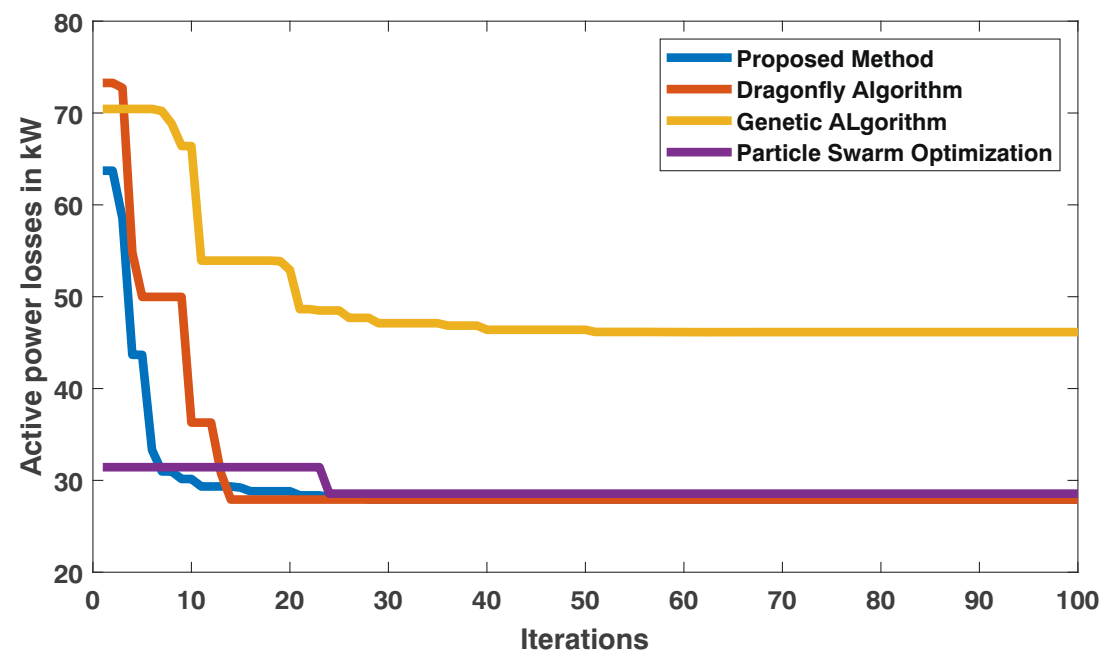


Fig. 12 Convergence

characteristics comparison

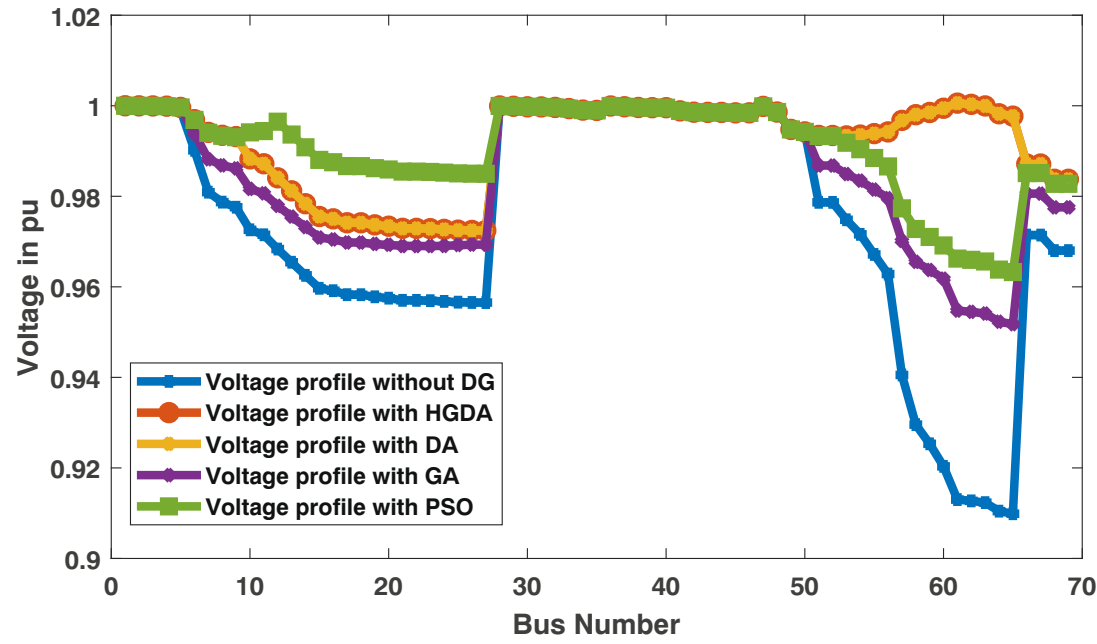

The performance of HGDA and DA are almost similar for optimal allocation of DG on PG\& E 69 bus distribution system if best simulation chosen. However, HGDA outperforms remaining all algorithms when tested in stochastic environment and it is necessary as all algorithms are stochastic in nature.

\section{Conclusions}

The integration of distributed generation into the distribution system is growing rapidly due to technical, economic, and environmental benefits. If DG units integrated to the system correctly, it will have a positive impact on the system; otherwise, it will have a negative impact on the system. It is necessary to develop an efficient algorithm for proper integration of DG units into the distribution system.

A new algorithm known as the hybrid genetic dragonfly algorithm was developed to find the optimal location and size of DG units by combining the genetic algorithm and the dragonfly algorithm. The optimization problem that deals with optimal placement of DG units has an objective function, which is loss minimization, and it also takes bus voltage limits and line thermal limits into account as constraints. Based on the simulation results, it was observed that the proposed algorithm performing well with a solution more near to global optimum point and also performs stochastically well with zero standard deviation.

The proposed method can assist distribution network operators in operating the network optimally in terms of active power losses while also operating the network at a lower cost. This DG placement problem can also be extended by considering emissions and reliability as objectives, as well as the impact of load modeling and also extended by considering the uncertainties in load and renewable energy.

\section{References}

1. Veeramsetty V, Chintham V, Kumar DMV (2020) Probabilistic locational marginal price computation in radial distribution system based on active power loss reduction. IET Generation, Transmis Distrib 14(12):2292

2. Rakesh R, VenkataPapana P, Keerthi S (2017) hybrid algorithm for optimal allocation of dg in radial distribution system. In: 2017 IEEE Region 10 Symposium (TENSYMP). IEEE, pp 1-5

3. Song Y, Wang G, Johns A, Wang P (1997) Distribution network reconfiguration for loss reduction using fuzzy controlled evolutionary programming. IEE Proceedings-Generation. Transm Distrib 144(4):345

4. Ghiasi M, Olamaei J (2016) Optimal capacitor placement to minimizing cost and power loss in tehran metro power distribution system using etap (a case study). Complexity 21(S2):483

5. Nagaballi S, Kale VS (2020) Pareto optimality and game theory approach for optimal deployment of $\mathrm{dg}$ in radial distribution system to improve techno-economic benefits. Applied Soft Computing. 106234

6. Almabsout EA, El-Sehiemy RA, An ONU, Bayat O (2020) A hybrid local search genetic algorithm for simultaneous placement of dg units and shunt capacitors in radial distribution systems. IEEE Access 8:54465

7. Samala RK, Kotapuri MR (2020) Optimal allocation of distributed generations using hybrid technique with fuzzy logic controller radial distribution system. SN Applied Sci 2(2):1

8. Truong KH, Nallagownden P, Elamvazuthi I, Vo DN (2020) An improved meta-heuristic method to maximize the penetration of distributed generation in radial distribution networks. Neural Comput Applic 32(14):10159

9. Selim A, Kamel S, Nasrat LS, Jurado F (2020) Voltage stability assessment of radial distribution systems including optimal allocation of distributed generators. International Journal of Interactive Multimedia \& Artificial Intelligence 6(1)

10. Jayasree M, Sreejaya P, Bindu G (2019) Multi-objective metaheuristic algorithm for optimal distributed generator placement and profit analysis. Technol Econ Smart Grids Sustain Energ 4(1):11

11. Jalili A, Taheri B (2020) Optimal sizing and sitting of distributed generations in power distribution networks using firefly algorithm. Technol Econ Smart Grids Sustain Energ 5(1):1

12. Manas M, Saikia BJ, Baruah DC (2018) Optimal distributed generator sizing and placement by analytical method and fuzzy 
expert system: a case study in tezpur university, India. Technol Econ Smart Grids Sustain Energ 3(1):1

13. Kim JO, Nam SW, Park S, Singh C (1998) Dispersed generation planning using improved hereford ranch algorithm. Electr Power Syst Res 47(1):47

14. Gandomkar M, Vakilian M, Ehsan M (2005) A genetic-based tabu search algorithm for optimal dg allocation in distribution network. Electric Power Comp Syst 33(12):1351

15. Haesen E, Driesen J, Belmans R (2007) Robust planning methodology for integration of stochastic generators in distribution grids. IET Renewable power generation 1(1):25

16. Singh R, Goswami S (2009) Optimum siting and sizing of distributed generations in radial and networked systems. Electric Power Components and Systems 37(2):127

17. Singh D, Singh D, Verma K (2009) Multiobjective optimization for dg planning with load models. IEEE transactions on power systems 24(1):427

18. Veeramsetty V, Lakshmi GN, Jayalaxmi A (2012) Optimal allocation and contingency analysis of embedded generation deployment in distribution network using genetic algorithm. In: 2012 International Conference on Computing, Electronics and Electrical Technologies (ICCEET). IEEE, pp 86-91

19. Prommee W, Ongsakul W (2011) Optimal multiple distributed generation placement in microgrid system by improved reinitialized social structures particle swarm optimization. European Transactions on Electrical Power 21(1):489

20. El-Zonkoly A (2011) Optimal placement of multi-distributed generation units including different load models using particle swarm optimisation. IET generation, transmission \& distribution $5(7): 760$

21. Wang L, Singh C (2008) Reliability-constrained optimum placement of reclosers and distributed generators in distribution networks using an ant colony system algorithm. IEEE Transactions on Systems, Man, and Cybernetics, Part C (Applications and Reviews) 38(6):757

22. Abu-Mouti FS, El-Hawary M (2011) Optimal distributed generation allocation and sizing in distribution systems via artificial bee colony algorithm. IEEE transactions on power delivery 26(4):2090

23. Rao RS, Ravindra K, Satish K, Narasimham S (2012) Power loss minimization in distribution system using network reconfiguration in the presence of distributed generation. IEEE Transactions on Power Systems 28(1):317

24. Ghosh S, Ghoshal SP, Ghosh S (2010) Optimal sizing and placement of distributed generation in a network system. International Journal of Electrical Power \& Energy Systems 32(8):849

25. Koutroumpezis G, Safigianni A (2010) Optimum allocation of the maximum possible distributed generation penetration in a distribution network. Electr Power Syst Res 80(12):1421

26. Mohd Ikhwan RR, Ridzuan1 M, Nur Nabihah MF, Farhanie N, Muhammad Adib ZR (2020) Reliabilitybased dg location using montecarlo simulation technique. SN Applied Sciences 2(145):742

27. Lakshmi GN, Jayalaxmi A, Veeramsetty V (2020) Optimal placement of distributed generation using firefly algorithm. In: IOP Conference Series: Materials Science and Engineering, vol 981, vol 981. IOP Publishing, p 042060
28. Fathi M, Ghiasi M (2019) Optimal dg placement to find optimal voltage profile considering minimum dg investment cost in smart neighborhood. Smart Cities 2(2):328

29. Goldberg DE, Holland JH (1988) Genetic algorithms and machine learning. Machine Learning 3(2):95

30. Deb K (1999) An introduction to genetic algorithms. Sadhana 24(4-5):293

31. Deb K, Deb D (2014) Analysing mutation schemes for realparameter genetic algorithms. International Journal of Artificial Intelligence and Soft Computing 4(1):1

32. Sudhakar AV, Karri C (2017) Bio inspired algorithms in power system operation: A review. In: 2017 International Conference on Recent Trends in Electrical, Electronics and Computing Technologies (ICRTEECT). IEEE, pp 113-119

33. Basetti V, Chandel AK, Subramanyam K (2018) Power system static state estimation using jade-adaptive differential evolution technique. Soft Comput 22(21):7157

34. Vedik B, Shiva C, Harish P (2020) Reverse harmonic load flow analysis using an evolutionary technique. SN Applied Sciences 2(9):1

35. Mirjalili S (2016) Dragonfly algorithm: a new meta-heuristic optimization technique for solving single-objective, discrete, and multi-objective problems. Neural Comput \& Applic 27(4):1053

36. Yang XS (2014) Nature-inspired optimization algorithms. Elsevier, Amsterdam

37. Juang CF (2004) A hybrid of genetic algorithm and particle swarm optimization for recurrent network design. IEEE Transactions on Systems, Man, and Cybernetics, Part B (Cybernetics) 34(2):997

38. KS SR, Murugan S (2017) Memory based hybrid dragonfly algorithm for numerical optimization problems. Expert Syst Appl 83:63

39. Shirmohammadi D, Hong H, Semlyen A, Luo G (1988) A compensation-based power flow method for weakly meshed distribution and transmission networks. IEEE Transactions on Power Systems 3(2):753

40. Wang Z, Chen F, Li J (2004) Implementing transformer nodal admittance matrices into backward/forward sweepbased power flow analysis for unbalanced radial distribution systems. IEEE Transactions on Power Systems 19(4):1831

41. Abdel-Akher M, Generation IET (2013) Voltage stability analysis of unbalanced distribution systems using backward/forward sweep load-flow analysis method with secant predictor. Transmission \& Distribution 7(3):309

42. Release M (2013) The mathworks. Inc., natick, massachusetts, United States 488

43. Veeramsetty V, Venkaiah C, Kumar DV (2017) Hybrid genetic dragonfly algorithm based optimal power flow for computing lmp at dg buses for reliability improvement. Energy Systems 1-49

44. Eberhart R, Kennedy J (1995) A new optimizer using particle swarm theory. In: Machine and Human Science 1995 MHS'95., Proceedings of the Sixth International Symposium on. IEEE, pp 39-43

45. Ryan TP (2011) Statistical methods for quality improvement. Wiley, Hoboken

Publisher's Note Springer Nature remains neutral with regard to jurisdictional claims in published maps and institutional affiliations. 